\title{
Traffic Sensing Methodology Combining Influence Line Theory and Computer Vision Techniques for Girder Bridges
}

\author{
Xudong Jian, ${ }^{1}$ Ye Xia ${ }^{(D)}{ }^{2}$ Jose A. Lozano-Galant, ${ }^{3}$ and Limin Sun ${ }^{1}$ \\ ${ }^{1}$ State Key Laboratory for Disaster Reduction in Civil Engineering, Tongji University, Shanghai 200092, China \\ ${ }^{2}$ Department of Bridge Engineering, Tongji University, Shanghai 200092, China \\ ${ }^{3}$ Department of Civil Engineering, University of Castilla-La Mancha, Ciudad Real 13071, Spain \\ Correspondence should be addressed to Ye Xia; yxia@tongji.edu.cn
}

Received 22 January 2019; Revised 8 March 2019; Accepted 7 May 2019; Published 27 May 2019

Guest Editor: Samir Mustapha

Copyright (C) 2019 Xudong Jian et al. This is an open access article distributed under the Creative Commons Attribution License, which permits unrestricted use, distribution, and reproduction in any medium, provided the original work is properly cited.

\begin{abstract}
Collecting the information of traffic load, especially heavy trucks, is crucial for bridge statistical analysis, safety evaluation, and maintenance strategies. This paper presents a traffic sensing methodology that combines a deep learning based computer vision technique with the influence line theory. Theoretical background and derivations are introduced from both aspects of structural analysis and computer vision techniques. In addition, to evaluate the effectiveness and accuracy of the proposed traffic sensing method through field tests, a systematic analysis is performed on a continuous box-girder bridge. The obtained results show that the proposed method can automatically identify the vehicle load and speed with promising efficiency and accuracy and most importantly cost-effectiveness. All these features make the proposed methodology a desirable bridge weigh-in-motion system, especially for bridges already equipped with structural health monitoring system.
\end{abstract}

\section{Introduction}

Modern bridges are mainly constructed for traffic purposes. Accordingly, collecting the information of traffic including vehicle weight, velocity, quantity, type, and spatiotemporal distribution, is crucial for bridge design refinement, safety evaluation, and maintenance strategies [1-3]. To this end, a number of studies on traffic information identification have been conducted. Among these methods, the bridge-weighin-motion (BWIM) technique is highlighted $[4,5]$.

The concepts behind BWIM techniques were initially proposed by Moses [6], who used an instrumented bridge as the weighing scale to estimate vehicle weights. Compared with other weigh-in-motion (WIM) techniques, such as the pavement-based WIM systems [7, 8], BWIM techniques are cost-efficient, durable, and unbiased as they are not impacted by repeated axle loads and do not require interrupting the traffic to cut the pavement. All these advantages have made BWIM a preferable tool to weigh vehicles, especially heavy trucks, attracting many follow-up research and engineering applications. Up to date, this research topic has progressed significantly in aspects as diverse as the identification results, such as time-history moving load identification [9-11], or the types of sensors, such as portable accelerometers [12].

One of the most simple and practical BWIM techniques verified by field tests is the gross vehicle weight (GVW). This identification method is based on the static influence line/surface theory, which is already applied by Moses in his earliest research [13]. However, key problems arise in obtaining accurate results when multiple vehicles cross the bridge deck simultaneously or move transversely [14]. In this scenario, combining supplemental vehicles position information and the influence surface instead of influence line might help to mitigate the problem. To position the vehicles on the bridge, traffic sensors such as radar, road tubes, and embedded axle detectors are recommended by Snyder et al. [15]. Lamentably, these sensors are too costly for its massive installation in actual structures. Alternatively, Xiao et al. [16] and Yamaguchi et al. [17] innovatively utilized the longitudinal ribs strains of an orthotropic steel bridge to detect the transverse position of vehicle axles. Unfortunately, concrete bridges without ribs are insensitive to single axle loads, making this method ineffective for this kind of structures. Yu et al. [18] proposed a novel BWIM algorithm that was 
able to identify the lateral position of a single vehicle on a bridge by using only seven strain gauges installed transversely at the bottom of the beams. That paper, however, admitted that identifying the presence of multiple-vehicle is still one of the main challenges faced by BWIM technology.

To address the multiple-vehicle presence challenge, using visual information is an innovative and feasible solution on the basis that a large number of bridges have been equipped with surveillance cameras for traffic monitoring of late years. In fact, the rich visual information recorded by the surveillance cameras enables obtaining the exact position of the vehicles on the bridge deck with nothing but a common webcam. Chen et al. [19] proposed an identification approach for the spatiotemporal distribution of traffic loads on bridges using the information from the pavement-based WIM and background subtraction technique. This approach relies on high quality video image, which limits its range of application. Another disadvantage of this method is the fact that it is nonsemantic, which means deep information contained in the video image, such as type and axle number of vehicles, is difficult to obtain. Similar problems also exist in studies aiming to detect vehicle axles using traditional computer vision techniques $[1,20]$.

In recent years, deep learning methods have dramatically improved the state of the art in visual object detection and recognition with amazing efficiency and robustness [21]. Inspired by the tremendous advance of computer vision techniques, this paper presents a traffic information identification methodology in combination with influence line theory and deep learning based computer vision techniques.

This paper is organized as follows. Firstly, the theoretical background of both aspects of structural analysis and computer vision techniques is presented. Next, field tests on a box-girder bridge were conducted to evaluate the proposed methodology in various aspects. Finally, both the advantages and the potential engineering applications of the methodology are discussed.

\section{Structural Analysis}

2.1. Bridge Response Analysis. One of the most concerning traffic information is vehicle weight. To estimate the vehicle weight, BWIM technology traditionally uses the bridge strains. Therefore, bridge structural strain analysis is essential in the process of vehicle weight identification.

Most BWIM systems are applied on girder bridges with small or medium span due to its structural simplicity. Compared with long-span bridges, middle-small span girder bridges perform linear elasticity under normal operation, making them ideal weighing scales to estimate vehicle weights. Moreover, load effects on such bridges are relatively simple, which can be expressed as follows:

$$
\begin{aligned}
& \varepsilon_{\text {bridge }}=\varepsilon_{\text {environment }}+\varepsilon_{\text {vehicle }} \\
& \varepsilon_{\text {vehicle }}=\varepsilon_{\text {dynamic }}+\varepsilon_{\text {static }}
\end{aligned}
$$

where $\varepsilon_{\text {bridge }}$ is the directly measured bridge strain; $\varepsilon_{\text {environment }}$ is the bridge strain caused by environmental factors, such as temperature, wind, slight earth pulse, and creep of concrete; $\varepsilon_{\text {vehicle }}$ is the bridge strain induced by vehicles, which includes dynamic $\varepsilon_{\text {dynamic }}$ and static $\varepsilon_{\text {static }}$ components.

According to the influence theory, the static component $\varepsilon_{\text {static }}$ is to be extracted from $\varepsilon_{\text {bridge }}$ by filtering $\varepsilon_{\text {environment }}$ and $\varepsilon_{\text {dynamic }}$ for the purpose of traffic load identification. In this paper, the filtering process is divided into two steps: (i) the $\varepsilon_{\text {bridge }}$ time-history curve is robustly smoothed to get $\varepsilon_{\text {environment }}$ and subtract $\varepsilon_{\text {environment }}$ from $\varepsilon_{\text {bridge }}$ to get $\varepsilon_{\text {vehicle }}$, and (ii) the $\varepsilon_{\text {vehicle }}$ time-history curve is smoothed to get the desired $\varepsilon_{\text {static }}$. The whole procedure is shown in Figure 1 for intuitive illustration.

To achieve the filtering process in time domain, a local regression algorithm named locally weighted scatterplot smoothing (LOWESS) is used. Chief attractions of this algorithm are the accuracy and convenience. It is not required to specify a global function of any form to fit a model to the data, only to fit segments of the data so that satisfactory local accuracy is achieved. According to Cleveland and Devlin [22], the basic principle of the LOWESS is expressed as follows.

First of all, the LOWESS belongs to the regression analysis, which aims to fit the mathematical relationship between two sequences $x_{i}$ and $y_{i}$. In this paper, $x_{i}$ is considered as the time sequence $t_{i}$, while $y_{i}$ is the bridge strain data sequence $\varepsilon_{i}$.

The LOWESS adopts the polynomial regression model, expression of which is [23]

$$
\begin{aligned}
\varepsilon_{i}=\beta_{0}+\beta_{1} t_{i}+\beta_{2} t_{i}^{2}+\cdots+\beta_{d} t_{i}^{d}+\varepsilon_{i}= & \sum_{j=0}^{d} \beta_{j} t_{i}^{j}+\theta_{i} \\
& (i=1,2, \ldots, n)
\end{aligned}
$$

where $\beta_{j}$ is the coefficient of the polynomial regression model, $d$ is the order of the polynomial, $\theta_{i}$ is the random error, and $n$ is the length of local sequence segment. For LOWESS, taking $d=2$ should almost always provide adequate smooth and computational efficiency.

To get appropriate coefficient $\widehat{\beta}_{j}\left(t_{i}\right)$ of the polynomial, the LOWESS chooses weighted least squares estimate method, which means $\widehat{\beta}_{j}\left(t_{i}\right)$ are the values that minimize the following function:

$$
E=\sum_{k=1}^{n} w_{k}\left(t_{i}\right)\left(\varepsilon_{k}-\beta_{0}-\beta_{1} t_{k}-\cdots-\beta_{d} t_{k}^{d}\right)^{2}
$$

where $w_{k}\left(t_{i}\right)$ are weights defined for all $t_{k}(k=1, \ldots, n)$. The tri-cube weight function is adopted to provide adequate smooth results.

Thus $\widehat{\beta}_{j}\left(t_{i}\right)$ can be obtained by

$$
\frac{\partial E}{\partial \beta}=0
$$

Finally, smoothing results are

$$
\widehat{\varepsilon}_{i}==\sum_{j=0}^{d} \widehat{\beta}_{j}\left(t_{i}\right) t_{i}^{j} \quad(i=1,2, \ldots, n)
$$

where $\widehat{\varepsilon}_{i}$ is the smoothed strain sequence. 


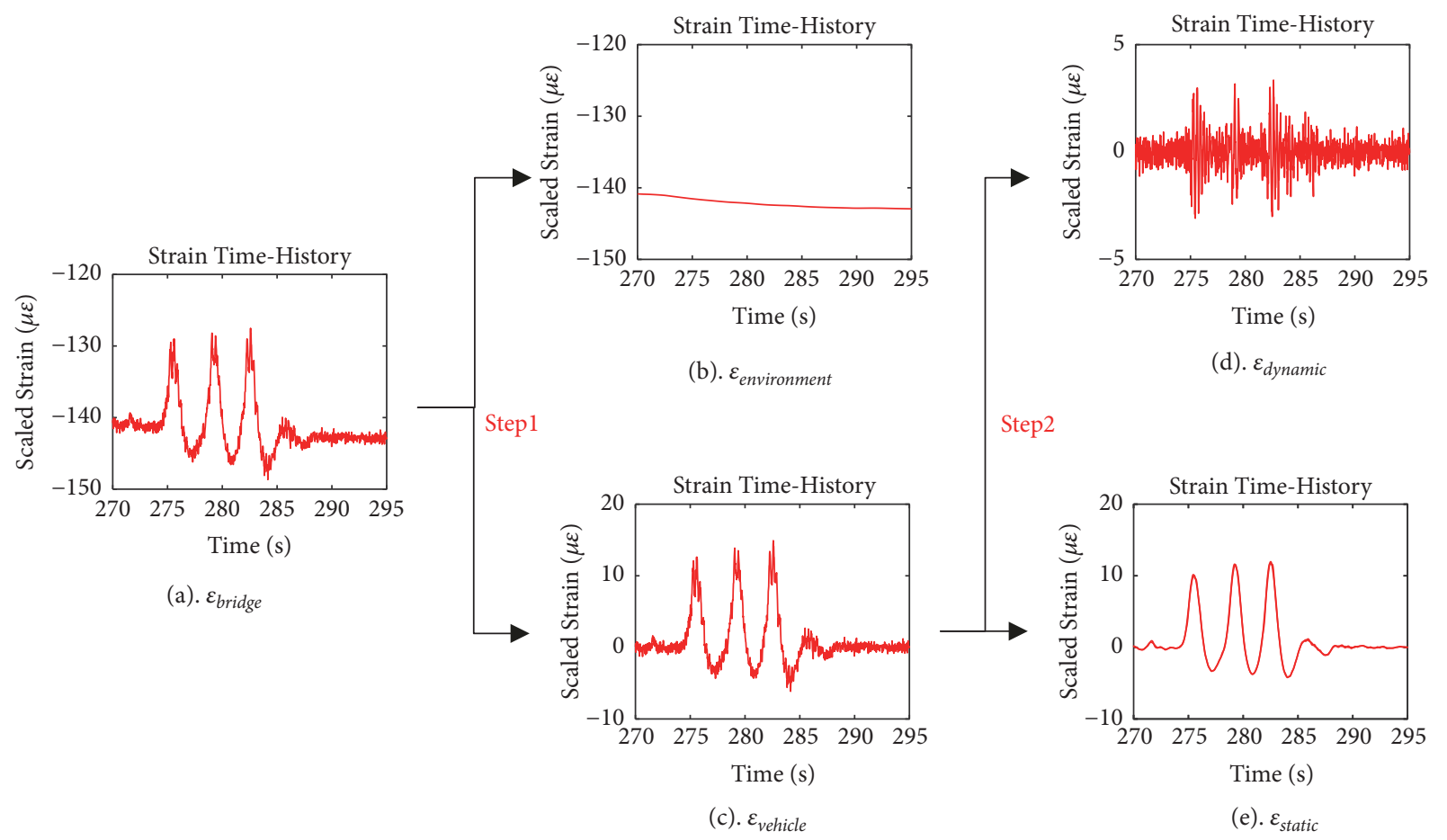

Figure 1: Procedure of the vehicle induced static strain extraction.

After preselecting $d$, the order of the polynomial, and $w_{k}\left(t_{i}\right)$, the weight function, the only parameter left to be defined, is the length of local sequence segment, $n$. This parameter can be chosen on the basis of the data properties. In this paper, $n$ is selected as 50 when smoothing the $\varepsilon_{\text {vehicle }}$ time-history curve. Because the longest vibration period of girder bridges is less than 1s, and the sampling frequency of strain sensors in this research is fixed at $50 \mathrm{~Hz}$, which means that 50 data points are recorded per minute. Choosing the length of the data sequence segment as 50 for locally smoothing is enough to filter the $\varepsilon_{\text {dynamic }}$ from the $\varepsilon_{\text {vehicle }}$ hence. Similarly, $n=500$ can be assumed for smoothing the $\varepsilon_{\text {bridge }}$ time-history curve when a vehicle crosses a bridge with small or medium span. In these cases, the frequency is usually within 10s.

The LOWESS algorithm is capable of smoothing the $\varepsilon_{\text {vehicle }}$ time-history curve to get the desired $\varepsilon_{\text {static }}$. However, using LOWESS to smoothing $\varepsilon_{\text {bridge }}$ might not be satisfactory enough. Compared with $\varepsilon_{\text {dynamic }}$, strain variation caused by vehicle weight is much more significant. Thus, the apparent peaks will distort the smoothed results as shown in Figure 2.

To prevent seriously deviant data from distorting the smoothed results, robust locally weighted regression (RLOWESS) algorithm was proposed on the basis of LOWESS [24]. Based on the size of the residual $e_{k}=\widehat{\varepsilon}_{k}-\varepsilon_{k}$, a different set of weights, $\delta_{k} \cdot w_{k}\left(t_{i}\right)$, is defined for each $\left(t_{i}, \varepsilon_{i}\right)$ as

$$
\delta_{k}= \begin{cases}{\left[1-\left(\frac{e_{k}}{6 s}\right)^{2}\right]^{2},} & \text { for }\left|\frac{e_{k}}{6 s}\right|<1 \\ 0, & \text { for }\left|\frac{e_{k}}{6 s}\right| \geq 1\end{cases}
$$

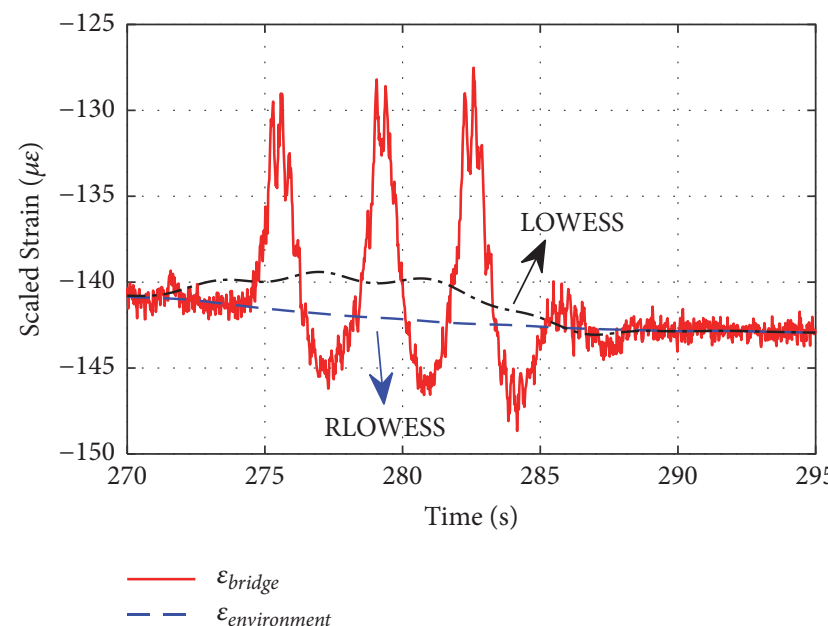

FIGURE 2: Normal strain signal collected from the web of a concrete box girder bridge.

where $\delta_{k}$ is the robust factor of weights, $e_{k}=\widehat{\varepsilon}_{k}-\varepsilon_{k}$ is the smoothing residual, and $s$ is the median of the $\left|e_{k}\right|$. By introducing $\delta_{k}$, large residuals result in small weights and small residuals result in large weights. In this way, distortion produced by seriously deviant data points can be effectively mitigated as shown in Figure 2.

2.2. Influence Line Calibration. Bridge influence lines can be used to weigh vehicles and they are vital tools for BWIM analysis. In fact, obtaining an adequate accuracy of the influence line is critical for the BWIM system to achieve 


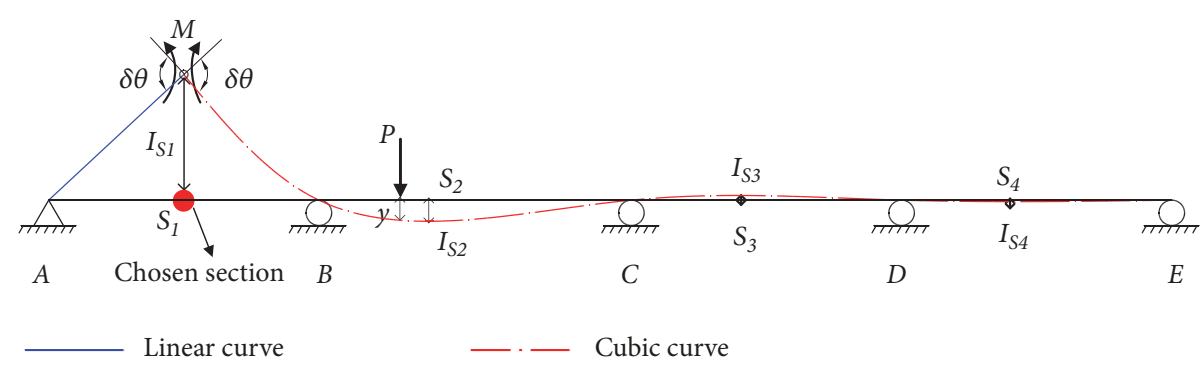

FIGURE 3: Diagram of the kinematic method aiming to obtain the influence line of section $S_{1}$.

convincing results. According to previous studies on BWIM, there are two methods to obtain the influence line of a bridge. One is the theoretical simulation method $[6,25,26]$, and the other is the calibration method carried out in field tests $[8,27]$.

Apparently, numerical simulation is unable to fully reproduce the mechanical behavior of a real bridge. To fill this gap, a method fitting strain influence line with measured strain data from field calibration tests is presented in this paper. The method includes the following two steps.

Step 1 (theoretically derive the shape of the influence line). In the first step, the theoretical shape of the strain influence line of the analyzed girder bridge is obtained. According to the structural mechanics, one of the most common methods to obtain the influence line of a chosen beam section is the kinematic one [28].

Normal strain of the chosen bridge cross-section is used to weigh vehicles in this work. As it is well known, the EulerBernoulli beam theory states that the normal strain of the chosen cross-section of a beam under vertical loads is proportional to its bending moment. According to Timoshenko and Gere [29], the proportional relationship can be expressed as

$$
\varepsilon=\frac{M y}{E I}
$$

where $\varepsilon$ is the normal strain of a point on the chosen beam cross-section, $M$ is the bending moment at that cross-section, $y$ is the distance between the point and the neutral axis of the cross-section, $E$ is the elastic modulus of the beam material, and $I$ is the moment of inertia of the cross-section.

Equation (8) indicates that, for a fixed point on the chosen beam cross-section, the normal strain $\varepsilon$ of that point is proportional to the bending moment $M$ at the cross-section. Thus the shape of strain influence line of a fixed point is similar to that of the bending moment influence line at the chosen cross-section where the fixed point is located. In other words, it can be said that the two influence lines are scaled.

To illustrate the kinematic method a four-span continuous beam presented in Figure 3 with nodes from $\mathrm{A}$ to $\mathrm{E}$ is considered. This method assumes that an element of the beam at the chosen cross-section, like $S_{1}$ section in Figure 3, is replaced with an ideal hinge. It allows relative rotation between the two portions of the beam and a system with one degree of freedom is obtained in this manner. If a load $P$ is applied at any point on the movable system, for equilibrium, a pair of two equal and opposite bending moments $M$ is needed at the hinge. Meanwhile, virtual displacement of the movable system will be produced by the loads. For the left movable portion $A S_{1}$, the displacement curve is linear, and, for the right structure portion $S_{1}-E$, the displacement curve is a cubic [29], as shown in Figure 3.

According to the principle of virtual work, the sum of corresponding virtual work of load $P$ and the couple $M$ equates zero, that is

$$
\begin{aligned}
M \cdot \delta \theta-P \cdot y & =0 \longrightarrow \\
M & =P \cdot \frac{y}{\delta \theta}
\end{aligned}
$$

where $\delta \theta$ is the total angular displacement between the two parts of the beam and $y$ is the vertical displacement of the point where load $\mathrm{P}$ is applied. Thus $y / \delta \theta$ refers to the influence coefficients for bending moment at the chosen section $S_{1}$, and the diagram of structural displacement has the shape of the influence line.

Step 2 (calibrating the derived influence line with field tests data). In the second step, numerical values of the strain influence line are calibrated from field test data. Figure 3 illustrates how the influence line can be numerically fitted after introducing the real measurements $(I)$ obtained in a field test at points $S_{1}, S_{2}, S_{3}$, and $S_{4}$ (that is to say $I_{S 1}, I_{S 2}, I_{S 3}$, and $\left.I_{S 4}\right)$.

The calibration approach begins with arranging a calibration truck with known weight to cross the instrumented bridge for several times, as Figure 4(a) shows.

Since bridges are usually long relative to the spacing of vehicle axles, gross vehicle weight is more important than individual axle load [30]. Besides, for the linear elastic structures, the mechanics principle of superposition works. Considering this, the vehicle load can be simplified as a concentrated load $P$, which is written as

$$
P=W \cdot g
$$

where $W$ is the vehicle weight, and $g$ is the gravitational acceleration.

According to the influence line theory, there will be an extreme on the strain time history curve recorded by a fixed strain sensor when a moving load passes a bridge span. For a four-span continuous girder bridge passed by the 


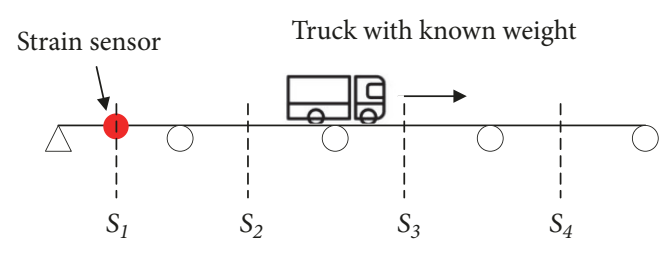

(a) Diagram of field calibration tests

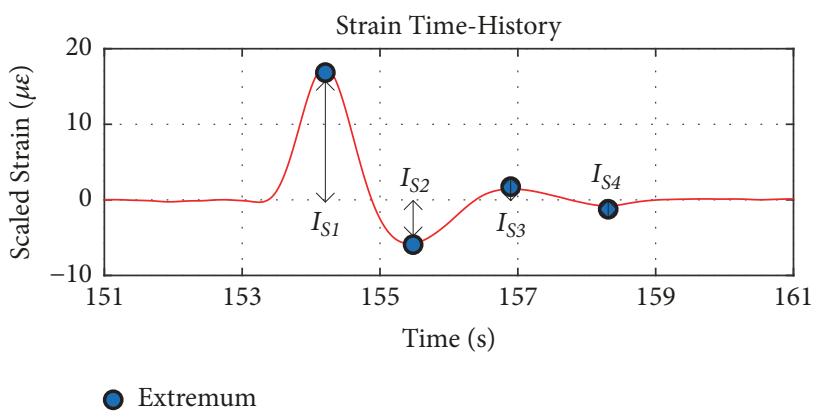

(b) Strain time history collected in tests

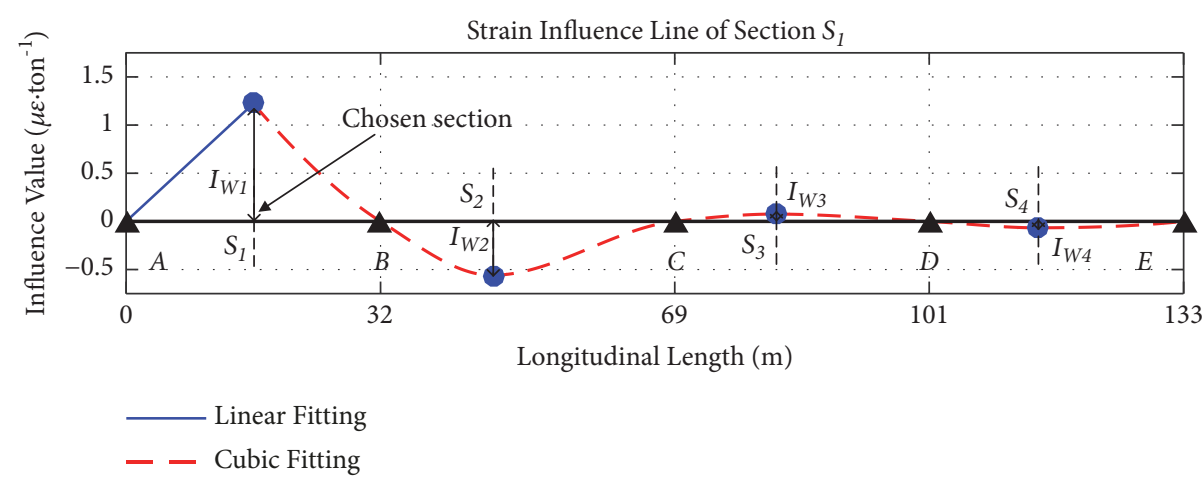

(c) Calibrated influence line of the chosen section

FIGURE 4: Procedure of the influence line calibration.

calibration truck, the $\varepsilon_{\text {static }}$ time-history curve of a fixed point on bridge has four extremes, as shown in Figure 4(b). The deflections $I_{s 1}, I_{s 2}, I_{s 3}$, and $I_{s 4}$ occur when the calibration truck passes cross-section $S_{1}, S_{2}, S_{3}$, and $S_{4}$. Then it is feasible to numerically fit the strain influence line of a desired point on the chosen section with nine points, $\mathrm{A} \sim \mathrm{E}$ and $S_{1} \sim S_{4}$ in Figure 3, of which the coordinates are determined.

Finally, the strain influence line is normalized to obtain the direct relationship between vehicle weight and bridge for BWIM application convenience. The normalization equation is as follows:

$$
I_{W}=\frac{I_{S}}{W}
$$

where $I_{W}$ is the static strain caused by per unit vehicle weight, and $I_{S}$ is the obtained value of the strain influence line. An example of calibrated strain influence line is shown in Figure 4(c). In this figure, four static strain values per unit vehicle weight $\left(I_{w 1}, I_{w 2}, I_{w 3}\right.$, and $\left.I_{w 4}\right)$ are considered. As discussed previously, the polynomial order for fitting purposes depends on the order of the displacement curve.

This calibration procedure of influence lines has a number of advantages, such as low calculation, operation simplicity, and no need to close traffic, enabling convenient recalibration if the mechanical performances of the instrumented bridge change [31]. However, it is noteworthy that the usage of influence line, instead of influence surface [32], is a simplification for real bridges, because vehicles may move transversely on bridge. But this simplification is still acceptable under the assumption that heavy trucks this research focuses on seldom change the traffic lane when crossing the bridge.

\section{Computer Vision Technique}

3.1. Deep Learning Approach. Convolution neural network (CNN) is one of the most notable deep learning approaches employed for object detection, classification, and segmentation tasks [33]. Here, learning means that CNN automatically learns useful features from the training data and distinguishes the target object and the others based on these features. Actually, that is how humans recognize objects. The CNN is therefore classified as artificial intelligence (AI) method. The learning ability is a qualitative leap over traditional manual feature extraction methods and can thus drastically reduce the workload of operation. Besides, the intelligent character also improves the robustness and generalization capacity because of the invariance to complex background, geometric distortion, and illumination.

Due to such advantages, new $\mathrm{CNN}$ based computer vision algorithms with better performance have been unceasingly proposed, and most of them are open source. This research applies the most advanced algorithm named YOLO V3 to fulfill the vehicle recognition tasks for its multi-scale and deeper feature extraction capacity as well as the fastest recognition speed up to date [34]. The application procedure has the following three steps.

Step 1 (preparing training data sets). As stated previously, CNN based computer vision algorithms will not work without effective training. Thus training data sets need to be prepared for the YOLO V3 algorithm at first. The preparatory work includes singling out segment of videos in which vehicles exist and manually labelling cars, trucks, and wheels 




(a) Manual labeling

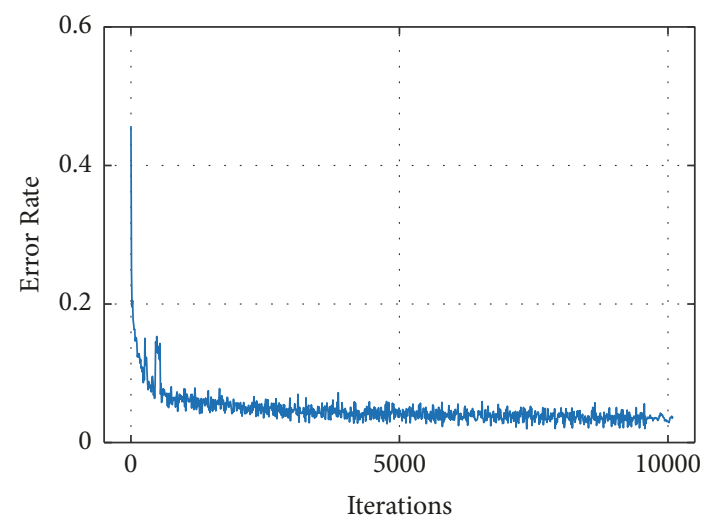

(b) Error curve in the training process

FIGURE 5: Preparation procedure of YOLO V3 algorithm.

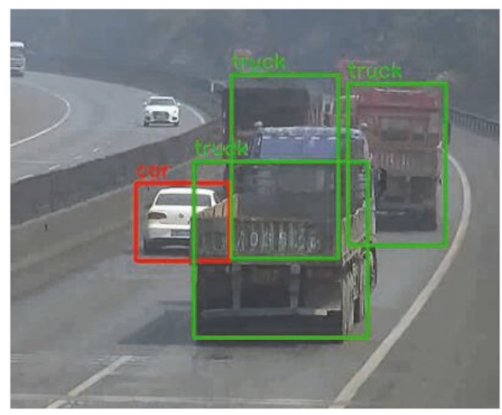

(a) Multi-vehicle overlap

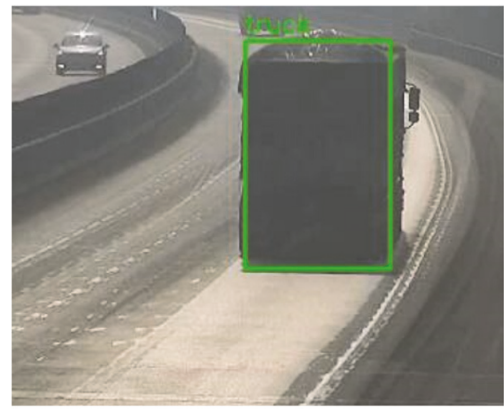

(b) Illumination change

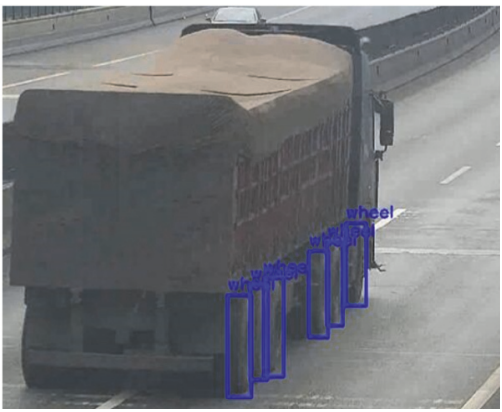

(c) Axle segmentation

FIGURE 6: Vehicle recognition results for different scenarios.

in every video frame, as shown in Figure 5(a). In this paper, a total of 1000 video images with the same camera visual angle are selected as the training set and different types of objects including cars, trucks, and wheels are labelled in each image.

Step 2 (training CNN of YOLO V3). CNN is essentially a set of weight coefficients capable of recognize objects using the pixel data of an image. Errors are inevitable in the process of recognition, and training $\mathrm{CNN}$ intends to obtain the optimal weight coefficients that minimizes the errors. To that end, the gradient descent method [33] is used in this optimization problem. This technique states

$$
\frac{\partial e}{\partial w_{i}} \leq r
$$

where $e$ is the recognition error, $w_{i}$ are the $\mathrm{CNN}$ weights, and $r$ is the convergence threshold.

Numerical iteration is required to achieve (12), and the iteration process is shown in Figure 5(b). In this research, iteration times are set to 10000 in the six-hour training process accelerated by a NVIDIA $1080 \mathrm{Ti}$ GPU.

Step 3 (applying YOLO V3). After a well-trained CNN is obtained, the YOLO V3 is used to recognize vehicles in real time. Recognition results in this research were quite satisfactory in the different scenarios shown in Figure 6. It is remarkable that the closely spaced wheels are successfully recognized as shown in Figure 6(c), proving the splendid recognition capability of the YOLO V3 algorithm. The recognized pixel coordinates of the detection box are collected for further vehicle positioning tasks.

Vehicle overlap and insufficient illumination might produce inevitable recognition errors. Diversifying neural network training sets and utilizing infrared camera at dark night will help to improve the recognition accuracy.

3.2. Coordinate Transformation. After the successful recognition by YOLO V3 algorithm, precise position of vehicles has to be determined. To address this problem, coordinate systems are established as illustrated in Figure 7 [35] and a coordinate transformation method is proposed in this work. There are two coordinate systems in the process of coordinate transformation, namely, camera pixel coordinate in the video image as shown in Figure 7(a) and space coordinate in the real world as shown in Figure 7(b). The relations between them are shown in Figures 7(c) and 7(d), respectively, where the parameters with same marks are equal. Based on the relations, the spatial coordinate of a point $P^{\prime}\left(x^{\prime}, y^{\prime}\right)$ on pixel plane can be transferred into $P(x, y, z)$ as follows:

$$
\begin{aligned}
& x=x^{\prime} \cdot t \\
& y=y^{\prime} \cdot t \\
& z=f \cdot t
\end{aligned}
$$




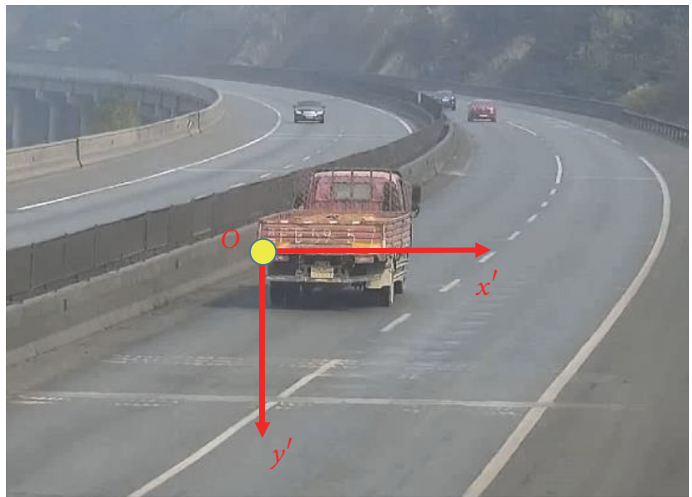

(a) Camera pixel coordinate system

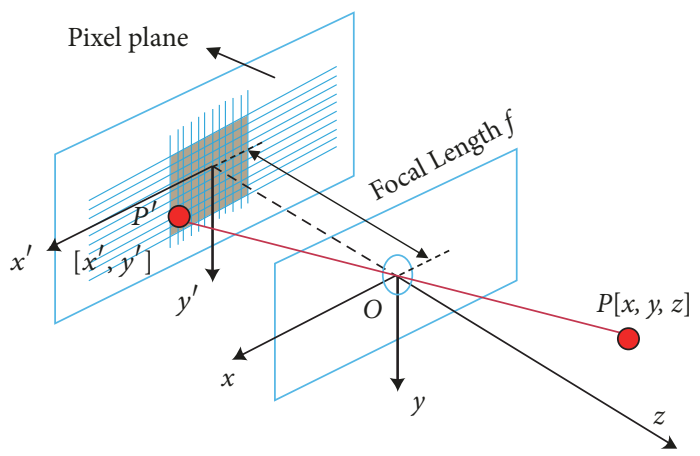

$P$ : Observed Object

O: Optical Center

$P^{\prime}$ : Image of the Object

(c) Camera imaging spatial model

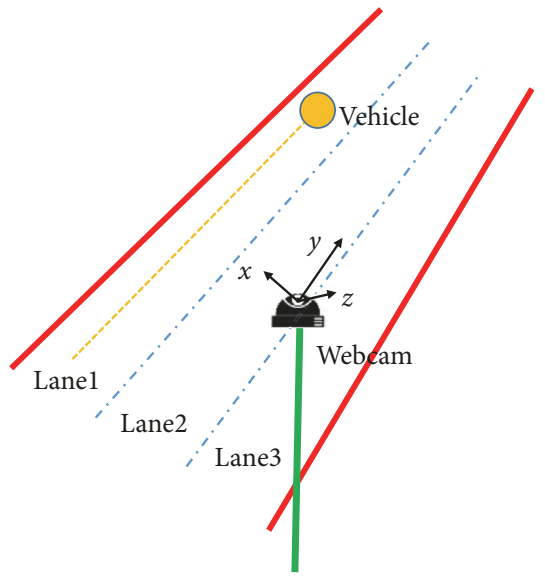

(b) Space coordinate system

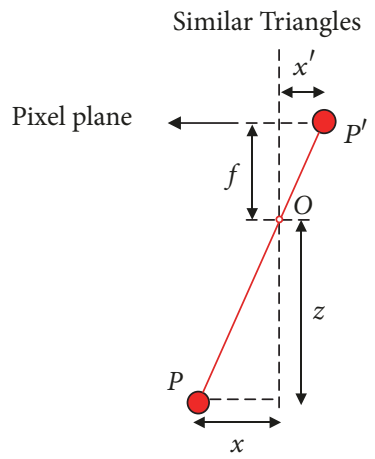

(d) Camera imaging planar model

FIGURE 7: Coordinate systems.

where $f$ is the focal length of the camera and $t$ is the similarity coefficient between the two similar triangles.

In the space coordinate, the bridge deck can be considered as a spatial plane represented by the following equation:

$$
A x+B y+C z+D=0
$$

where $x, y, z$ are the spatial coordinates of the observed object such as a truck, and $A, B, C, D$ are unknown parameters determining the bridge deck plane equation. If the bridge slope is negligible, which is the usual case, $x$ and $y$ directly determine position of vehicles on the bridge deck. Then vehicle coordinates on the deck are attainable after obtaining $A, B, C$ and $D$.

Instinctively, both location and orientation of the webcam are needed to obtain parameters $A, B, C$ and $D$. However, a number of field conditions, such as heavy traffic flow, make it difficult to obtain this information. To solve this problem, this paper proposes a method to obtain $A, B, C, D$ directly from the video image without knowing the camera location and/or its orientation. The proposed method only needs two lines of equal space length in the image. For example, lines $P_{1}{ }^{\prime} P_{2}{ }^{\prime}$ and $P_{3}{ }^{\prime} P_{4}{ }^{\prime}$ in Figure 8 have the equal length of $3.75 \mathrm{~m}$. The coordinates of their endpoints can be measured directly from the image.

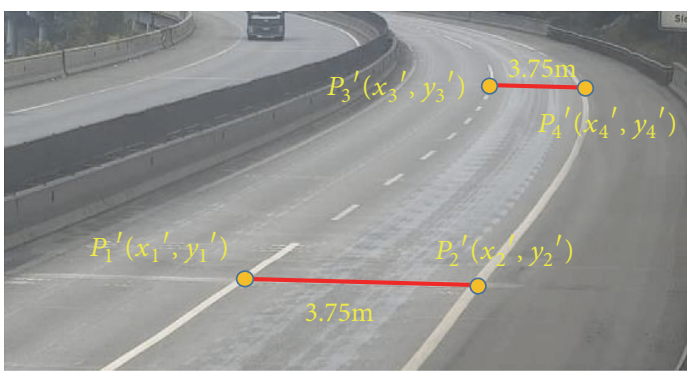

Figure 8: Diagram for $A, B, C, D$ calculation from two reference lines $P_{1}^{\prime}-P_{2}^{\prime}$ and $P_{3}{ }^{\prime}-P_{4}{ }^{\prime}$.

According to Figure 8, the relations between both lines can be written as follows:

$$
\begin{aligned}
\Delta x_{1} & =x_{1}{ }^{\prime}-x_{2}{ }^{\prime}, \\
\Delta y_{1} & =y_{1}{ }^{\prime}-y_{2}{ }^{\prime} \\
\Delta x_{2} & =x_{3}{ }^{\prime}-x_{4}{ }^{\prime}, \\
\Delta y_{2} & =y_{3}{ }^{\prime}-y_{4}{ }^{\prime} \\
\sqrt{\Delta x_{1}{ }^{2}+\Delta y_{1}{ }^{2}} \cdot t_{1} & =\sqrt{\Delta x_{2}{ }^{2}+\Delta y_{2}{ }^{2}} \cdot t_{2}=L
\end{aligned}
$$




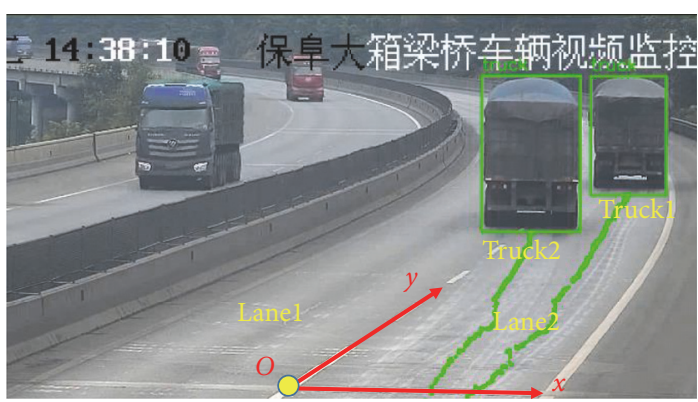

(a) Trajectory in image

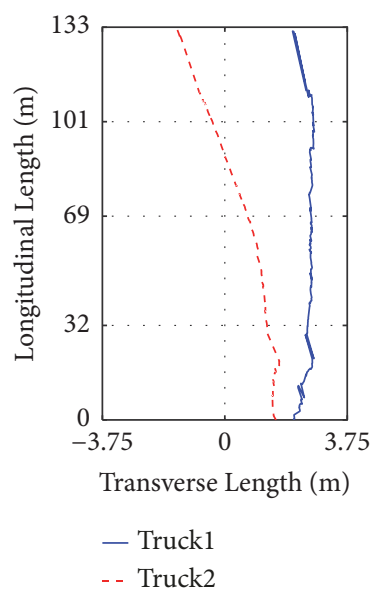

(b) Trajectory on bridge deck

FIGURE 9: Recognized vehicle trajectory.

where $\left(x_{1}{ }^{\prime}, y_{1}{ }^{\prime}\right),\left(x_{2}{ }^{\prime}, y_{2}{ }^{\prime}\right),\left(x_{3}{ }^{\prime}, y_{3}{ }^{\prime}\right)$, and $\left(x_{4}{ }^{\prime}, y_{4}{ }^{\prime}\right)$ are the coordinates of the endpoints $P_{1}{ }^{\prime}, P_{2}{ }^{\prime}, P_{3}{ }^{\prime}$, and $P_{4}{ }^{\prime}, t_{1}$ and $t_{2}$ are similarity coefficients of the lines, and $\mathrm{L}$ is the line length. With (15), parameter $t$ of the two lines can be separately calculated, then spatial coordinates of the four endpoints are obtained with (13). Substituting coordinates of the four endpoints of the two equal length lines into (14), the four unknowns $A, B, C$ and $D$ can be directly obtained.

The main advantage of this method is its simplicity, while the trade-off is the loss of accuracy assuming that parameter $t$ is equal for endpoints $P_{1}{ }^{\prime}$ and $P_{2}{ }^{\prime}$ as well as $P_{3}{ }^{\prime}$ and $P_{4}{ }^{\prime}$, which is true when the selected lines are far enough from the camera and the line length is short. Another noticeable error source comes from the camera imaging distortion, which is complicated and will not be discussed in this paper. Figure 9 depicts vehicle trajectory tracked by the aforementioned method.

\section{Field Tests}

4.1. Test Setup. In order to verify the applicability of the proposed traffic information identification methodology in real structures, field tests were conducted on a $32 m+37 m+32 m+32 m$ continuous concrete box girder bridge of Baoding-Duping Highway, China. There are three traffic lanes on the bridge in total, and each of them is $3.75 \mathrm{~m}$ wide. Among these traffic lines, lane 3 is an emergency lane where vehicles are prohibited to drive under normal conditions. The bridge is slightly curved with a bending radius of $2600 \mathrm{~m}$ and a central angle of $2.93^{\circ}$; thus the curvature effects are negligible in the analysis. A structural health monitoring system comprising a pavement-based WIM system, six resistancetype strain sensors, and a webcam is installed on this bridge. All the discussed information is shown in Figure 10.

In the field tests, the normal strain data were collected by the six resistance-type strain sensors mounted on the mid-span section of the first span and stored in an online server. Video recorded by the webcam is also available on line, providing a basis for long-term online application. Vehicle weight and velocity recognized by pavement-based WIM system are used as contrast to evaluate the accuracy of this proposed methodology.

4.2. Calibration Tests. First of all, field calibration tests as on the lanes presented in Figure 10(b) were implemented following the method mentioned in Section 2.2 up front. To do so, an ordinary truck weighing $14.86 \mathrm{t}$, as shown in Figure 11, was arranged to drive on the tested bridge for four times. Detailed test conditions are shown in Table 1. Lane3 was ignored for the calibration because it is an emergency lane where vehicles are prohibited to drive under normal conditions.

Figure 12 shows the influence line calibration results of the "S6" strain sensor in Figure 10(b). Differences between test1 and test 2 , as well as test 3 and test 4 , are slight, which verifies the feasibility and reliability of the proposed influence line calibration method. It is also observed that the influence value of traffic lanel is larger than that of lane2, as strain sensor " 56 " is located closer to lanel.

4.3. Strain Data Processing. Next, strain data collected by six resistance-type strain sensors, shown in Figure 10, is processed with the LOWESS algorithm mentioned in Section 2.1 of this paper. Taking a segment of the processed strain time history shown in Figure 13(a) as an example, an obvious linear relationship between data peak values of strain sensors mounted on the same box web is observed in Figure 13(b). The linear relationship confirms the plane section assumption of Euler-Bernoulli beam theory mentioned in (8) above and thus validates the effectiveness of the strain data processing method.

4.4. GVW Calculation. The gross vehicle weight (GVW) can be calculated by combining the calibrated strain influence line, the processed bridge strains, and the vehicle position. Basically, there are only three elementary vehicle distribution 


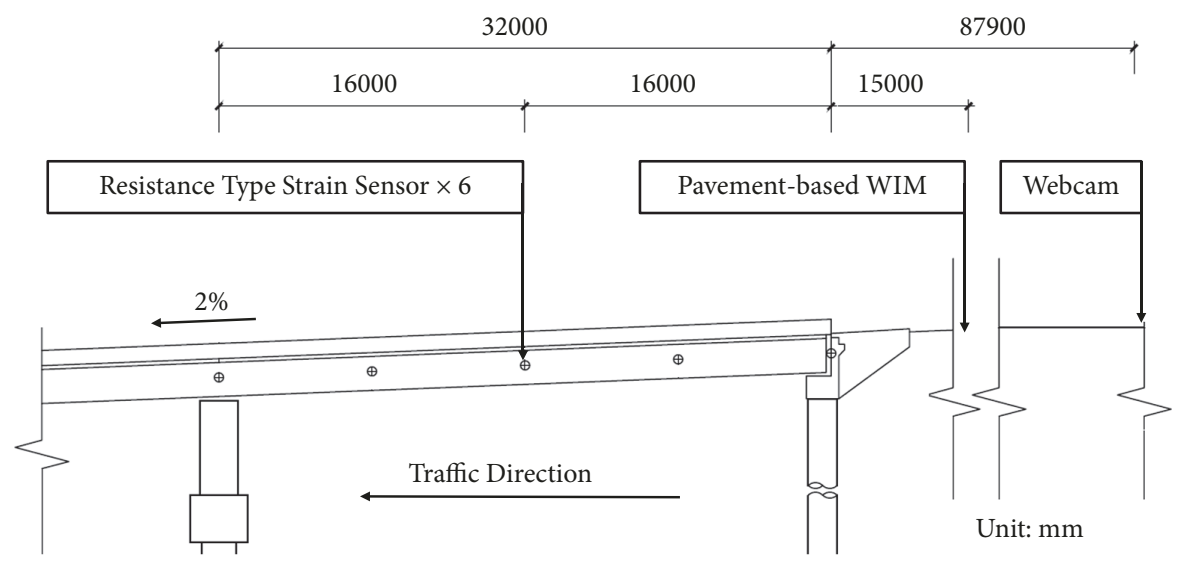

(a) Elevation view of the tested bridge

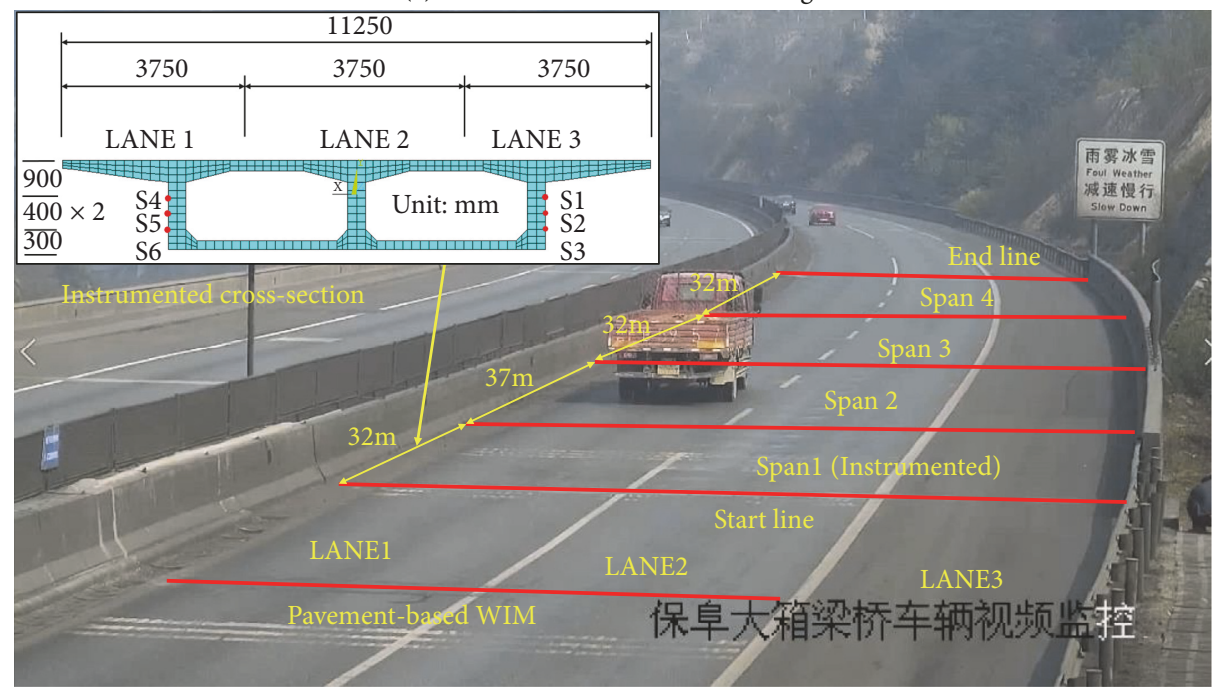

(b) Diagram of the tested bridge and the instrumented section

Figure 10: Bridge for field tests.

TABLE 1: Conditions of calibration tests.

\begin{tabular}{lcccc}
\hline & Test1 & Test2 & Test3 & \\
\hline Weight & $14.86 \mathrm{t}$ & $14.86 \mathrm{t}$ & $14.86 \mathrm{t}$ & $14.86 \mathrm{t}$ \\
Velocity & $60 \mathrm{~km} / \mathrm{h}$ & $80 \mathrm{~km} / \mathrm{h}$ & $60 \mathrm{~km} / \mathrm{h}$ & \\
Lane & Lane1 & Lane1 & Lane2 & Lane2 \\
\hline
\end{tabular}

scenarios presented in Figure 14. They are single vehicle in Figure 14(a), one-by-one vehicles on the same lane in Figure 14(b), and side-by-side vehicles on different lanes in Figure 14(c).

For the first single vehicle scenario, it is simple to calculate weight of the vehicle through the following equation:

$$
W=\frac{S^{\text {peak }}}{I^{\text {peak }}}
$$

where $S^{\text {peak }}$ is the peak value of vehicle induced static strain signal, $I^{\text {peak }}$ is the peak value of the calibrated strain influence line, and $W$ is the GVW of the vehicle.

For the second one-by-one vehicles scenario, GVW of the first front vehicle can still be calculated through (16). Then
GVW of the rear vehicles can be calculated after subtracting effects of the front vehicle whose GVW is already known. This process is written as

$$
W_{\text {rear }}=\frac{S_{\text {rear }}^{\text {peak }}-I_{\text {front }} \cdot W_{\text {front }}}{I_{\text {rear }}^{\text {peak }}}
$$

where $S_{\text {rear }}^{\text {peak }}$ is the peak value of the rear vehicle induced static strain signal, $I_{\text {front }}$ is the strain influence value related to the position of the front vehicle, which can be obtained with the aid of the aforementioned computer vision technique, $W_{\text {front }}$ is the GVW of the front vehicle calculated through (16), I rear is the peak value of the calibrated strain influence line of 
TABLE 2: Statistics of the relative errors compared with pavement-based WIM.

\begin{tabular}{|c|c|c|}
\hline Sensor & Mean of errors (\%) & Standard deviation of errors (\%) \\
\hline S1 & 35.2 & 37.4 \\
\hline S2 & -3.6 & 18.7 \\
\hline S3 & -2.8 & 20.8 \\
\hline S4 & 38.6 & 25.2 \\
\hline S5 & -1.8 & 12.4 \\
\hline S6 & -5.2 & 11.6 \\
\hline
\end{tabular}

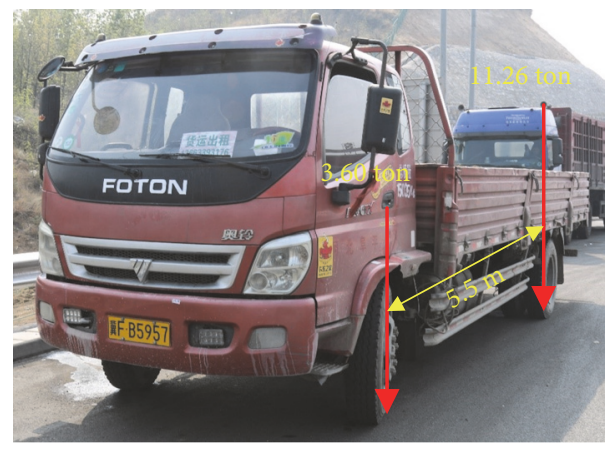

FIGURE 11: Calibration truck.

traffic lane where the rear vehicle drives, and $W_{\text {rear }}$ is the GVW of the rear vehicle.

It is important to highlight that using (16) to calculate the weight of the front vehicle in the one-by-one vehicle queue is not applicable to circumstances when the rear vehicle enters the bridge before the front vehicle passes the instrumented bridge cross-section, because $S^{\text {peak }}$ in (16) involves the effects of the rear vehicle under such circumstances. Fortunately, this problem does not exist in this research; for a sizeable safety margin, no less than $30 \mathrm{~m}$, between front and rear vehicles, is demanded when driving on highways in China. The distance between the instrumented cross-section and the start point of the bridge, however, is only $16 \mathrm{~m}$.

Challenge arises when two vehicles driving side by side, however. In this scenario, one strain signal peak corresponds to two indistinguishable vehicles, which makes the above GVW calculation methods ineffective.

Finally, a segment of 15 minutes' strain signal and video when there are no side-by-side trucks is analyzed. Cars are ignored and weights of a total of 61 trucks are calculated. Statistics of the relative errors compared with the results recognized by the pavement-based WIM system are listed in Table 2. Plots of the GVW results of the six sensors S1 S6 are also shown in Figure 15, in which each point corresponds to a vehicle. In this figure, the further away the point is from the baseline, the larger the error is.

According to the GVW calculation results, though errors of several vehicles are unpleasantly significant, accuracy of the rest is still acceptable, except results based on strain sensors named S1 and S4. Close distance to the section neutral axis of S1 and S4 explains their significant errors. Because, under the plane section assumption, the closer the strain sensor is to the neutral axis, the smaller its strain value, making the relative error larger in contrast. To avoid this problem, BWIM sensors should be installed far from the section neutral axis for higher accuracy.

4.5. Vehicle Velocity Calculation. Theoretically, instantaneous velocities of vehicles can be calculated through (18) with the recognized vehicle position in each video frame and fixed time interval between frames.

$$
v=\frac{\Delta S}{\Delta t}
$$

where $\mathrm{v}$ is the vehicle velocity and $\Delta S$ is the vehicle displacement within a period of time $\Delta t$.

However, calculated instantaneous velocities of vehicles appear to fluctuate drastically. Average vehicle velocity in three seconds, which means $\Delta t=3 \mathrm{~s}$, is calculated instead of instantaneously, and the calculation results are quite accurate as shown in Figure 16. The mean value of errors is $-0.8 \%$, the standard deviation of errors is $9.2 \%$, and the maximum value of errors is $23.1 \%$.

4.6. Vehicle Type and Axle Recognition. Identification of closely spaced axles, including tandem axles, is a key factor to ensure accurate classification of the passing vehicles. Realtime traffic characterization on a bridge is beneficial for asset managers and bridge owners because it provides statistical data about the configurations of the passing vehicles. The nothing-on-road (NOR) technique is generally utilized to obtain the information about the axles with sensors located underneath the bridge girder $[36,37]$.

As a supplement, this paper obtains information about vehicle type and number of axles with visual information provided by only a webcam. Figures 6(a) and 6(c) show that the well-trained YOLO V3 algorithm is capable of directly recognizing vehicle types and the number of axles similarly to humans. The vehicle type recognition accuracy is $100 \%$ and the axle recognition results of 61 trucks (including 6 trucks with 2 axles) and 50 cars in the field tests are shown in Figure 17, which is still quite satisfactory compared with the pavement-based WIM. Errors are inevitable because of vehicle overlap, limited visual angle of the webcam, and illumination conditions. For instance, if cars are obscured by trucks with large size or the illumination is rather dim, wheels of cars will thus not be recognized. For instance, if cars are obscured by trucks with larger size or the illumination is rather dim, cars wheels will thus not be recognized. That is the reason why the computer vision made mistakes. This problem 



(a) Results of test1 and test2


- - Test4

.

(b) Results of test 3 and test 4

FIGURE 12: Influence line calibration results.

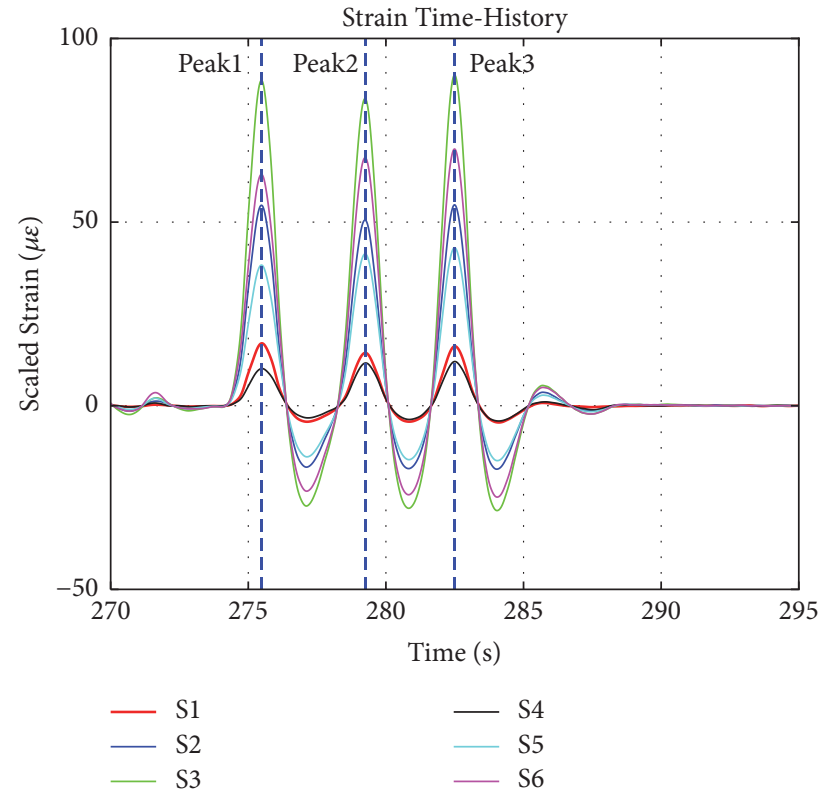

(a) Processed strain time-history curve

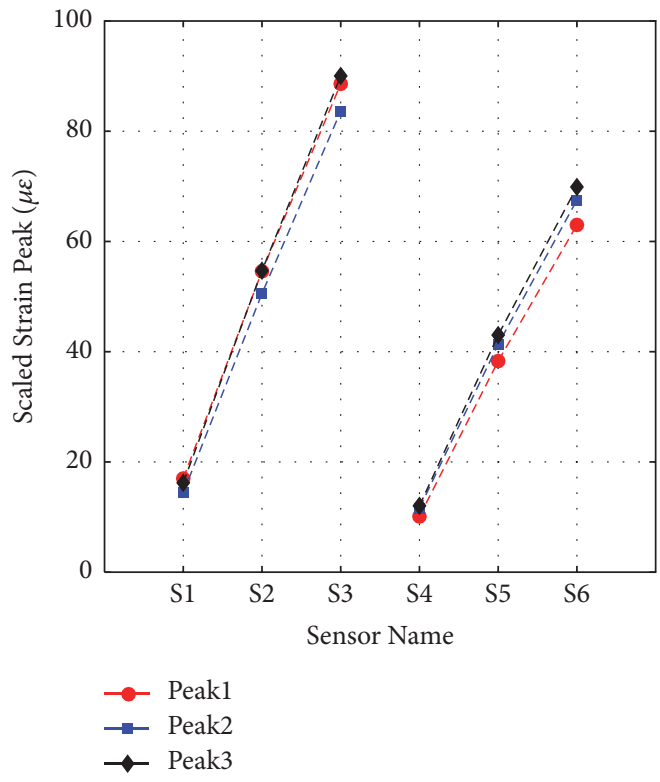

(b) Values of peaks

FIGURE 13: Strain data processing results.

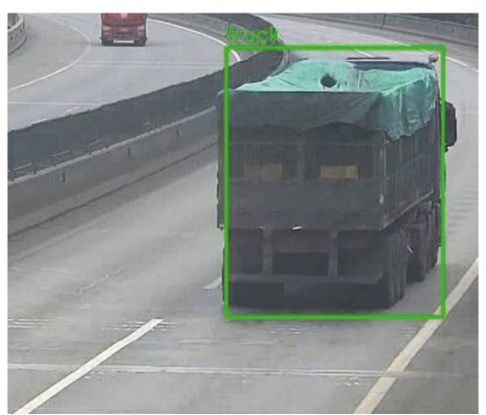

(a) Single vehicle

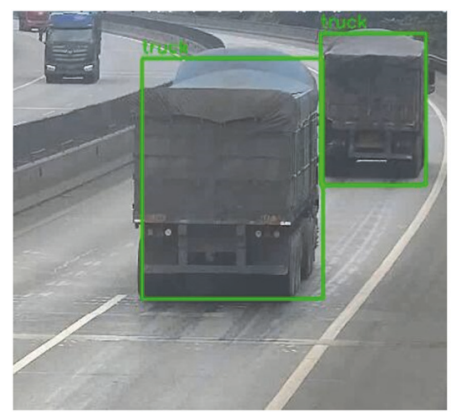

(b) One-by-one vehicles

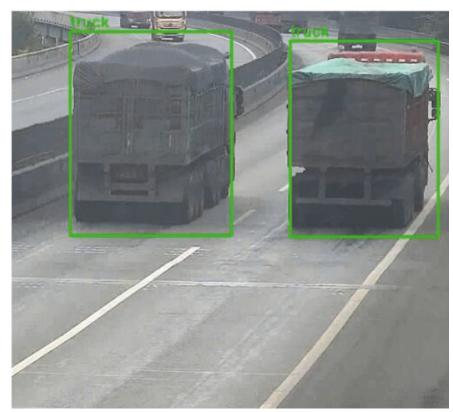

(c) Side-by-side vehicles

FIGURE 14: Scenarios of vehicle distribution on bridge. 


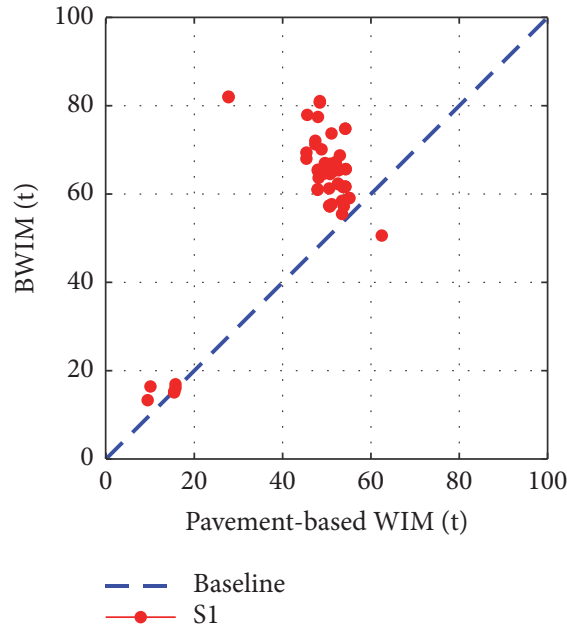

(a) GVW results based on S1 data

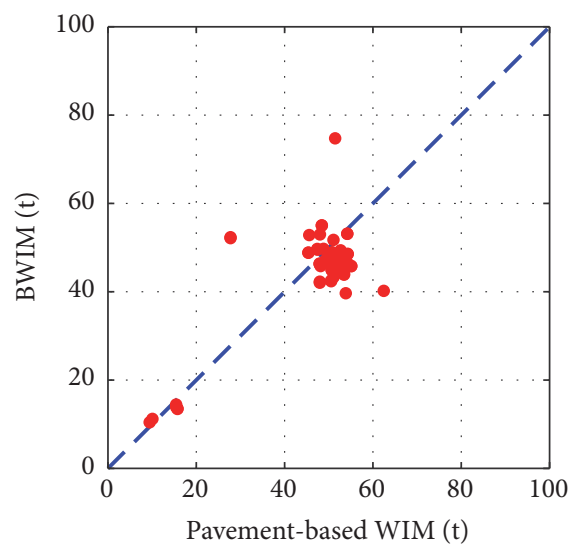

- - Baseline



(c) GVW results based on S3 data

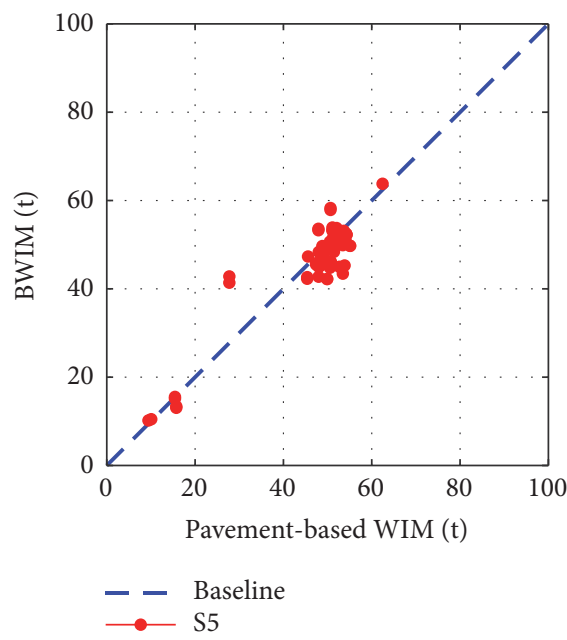

(e) GVW results based on S5 data

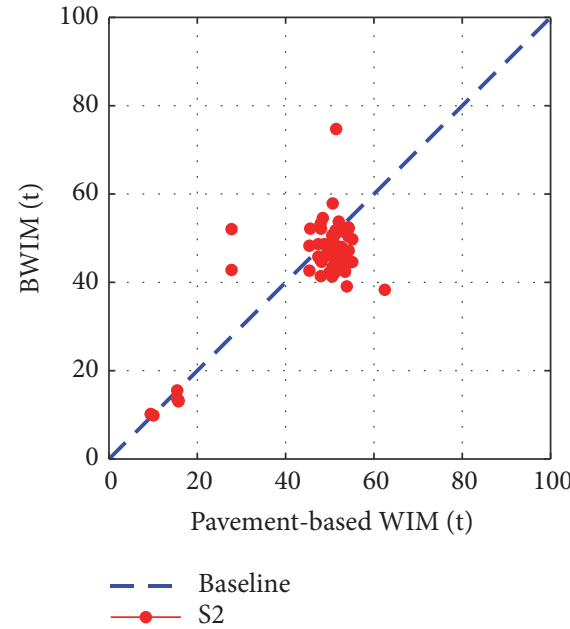

(b) GVW results based on S2 data

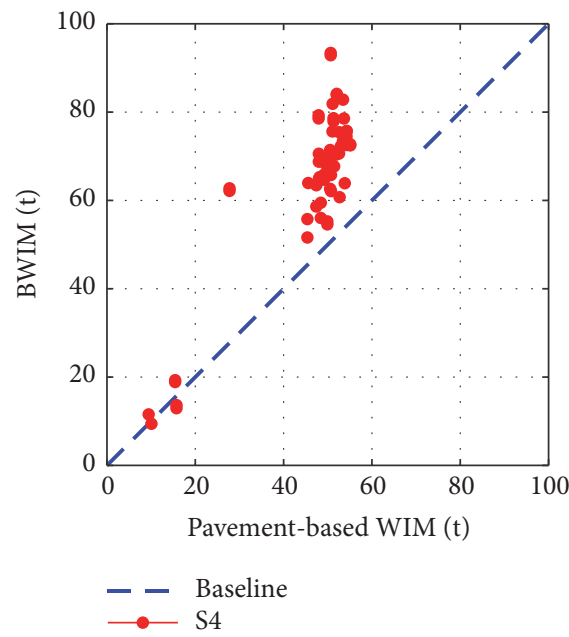

(d) GVW results based on S4 data



(f) GVW results based on S6 data

FIGURE 15: GVW calculation results for the six strain sensors S1 S6. 




FIGURE 16: Vehicle velocity results.

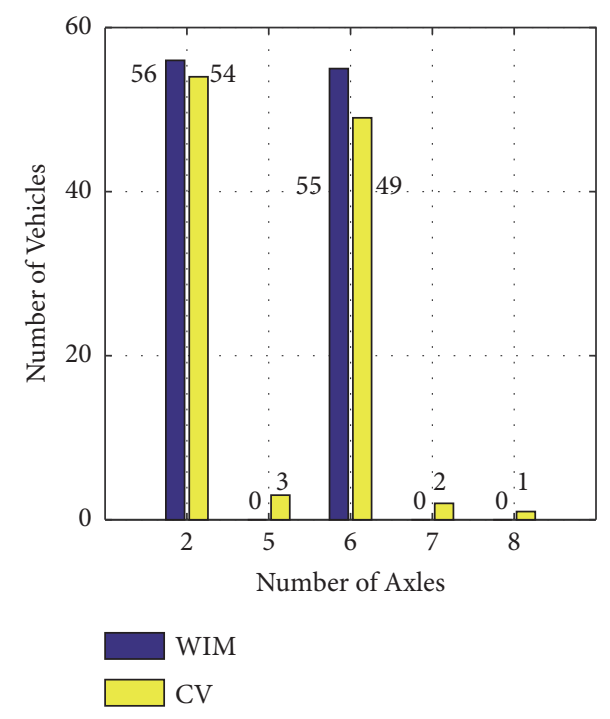

FIgURE 17: Axle recognition results.

did not appear in the pavement-based WIM as illustrated in Figure 17.

To prevent these errors, the visual angle of the webcam can be adjusted to observe the vehicle wheels more clearly. Another way to improve the quality of the vision is using an infrared camera to prevent dim illumination.

4.7. Error Analysis. Although the recognition accuracy is acceptable, error analysis is imperative for further improvement. To the author's knowledge, the following two reasons may account for calculation errors illustrated in Figure 18: (i) vehicle deviation from the traffic lane and (ii) vehicle positioning errors of the computer vision technique.

It is noted that vehicles on a bridge do not drive on the traffic lane strictly in some cases, but, in this research, only influence lines of traffic lanes are utilized. This assumption leads to significant errors when the vehicle deviates from the traffic lane severely as presented in Figure 18(a). To reduce this kind of error, the influence line can be substituted by the influence surface.

Another error source is inaccurate vehicle detection as shown in Figure 18(b), where multiple vehicles overlap in the image and leads to positioning errors. Particular labelling aiming at this phenomenon and diversifying the training sets for the deep neural network will help to mitigate the problem.

\section{Conclusions}

A traffic sensing methodology has been proposed in this paper in combination with influence line theory and computer vision technique. Field tests were conducted to evaluate the proposed methodology in various aspects. The main conclusions of this work might be listed as follows:

(1) The identification of vehicle positions, especially on transverse direction when passing a bridge, is quite critical to solve multiple-vehicle problem for BWIM systems. This paper introduces, for the first time, deep learning based computer vision technique to obtain the exact position of vehicles on bridges and successfully solves one-by-one vehicles scenario of multiple-vehicle problems for BWIM research with an average weighing error within 5\%.

(2) The time series smoothing algorithm, LOWESS, is an effective tool to extract static component from directly measured bridge responses. Then, influence line or influence surface of a real bridge can be easily calibrated for BWIM purpose.

(3) Verified by field tests, the deep learning based computer vision technique is highly stable and efficient to recognize vehicles on bridges in real time manner. Therefore, it is proven to be a promising technique for traffic sensing.

(4) The proposed traffic sensing methodology is capable of identifying vehicle weight, velocity, type, axle number, and time-spatial distribution on small and medium span girder bridges in a cost-effective way, especially for those bridges already equipped with structure health monitoring systems and surveillance cameras.

\section{Data Availability}

The video and testing data used to support the findings of this study are included within the article.

\section{Conflicts of Interest}

The authors declare that they have no conflicts of interest.

\section{Acknowledgments}

This paper is supported by the National Key R\&D Program of China (2017YFC1500605), Science and Technology Commission of Shanghai Municipality (17DZ1204300 and 


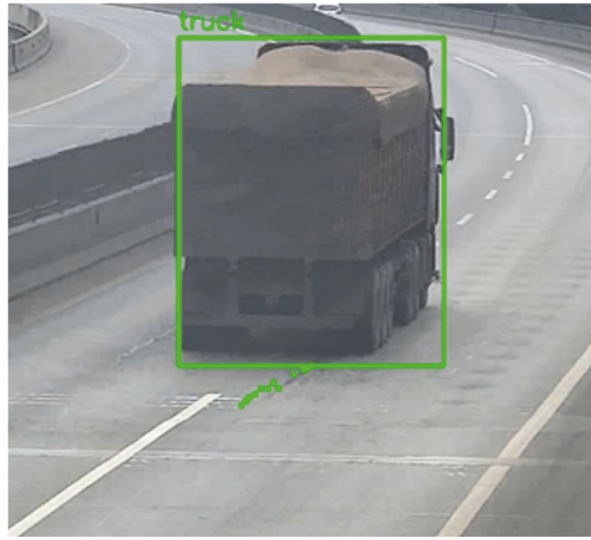

(a) Vehicle deviation from traffic lane

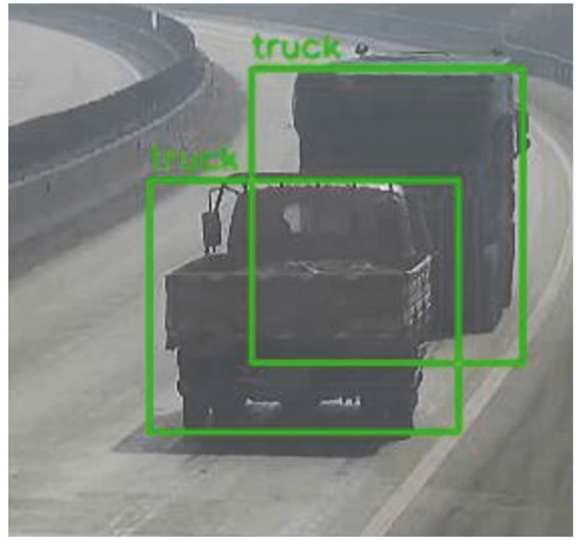

(b) Inaccurate vehicle detection

FIGURE 18: Major error sources.

18DZ1201203), and the Fundamental Research Funds for the Central Universities.

\section{References}

[1] R. Zaurin and F. N. Catbas, "Integration of computer imaging and sensor data for structural health monitoring of bridges," Smart Materials and Structures, vol. 19, no. 1, Article ID 015019, 2009.

[2] B. Wei, C. Zuo, X. He, L. Jiang, and T. Wang, "Effects of vertical ground motions on seismic vulnerabilities of a continuous track-bridge system of high-speed railway," Soil Dynamics and Earthquake Engineering, vol. 115, pp. 281-290, 2018.

[3] B. Wei, T. Yang, L. Jiang, and X. He, "Effects of uncertain characteristic periods of ground motions on seismic vulnerabilities of a continuous track-bridge system of high-speed railway," Bulletin of Earthquake Engineering, vol. 16, no. 9, pp. 3739-3769, 2018.

[4] Y. Yu, C. S. Cai, and L. Deng, "State-of-the-art review on bridge weigh-in-motion technology," Advances in Structural Engineering, vol. 19, no. 9, pp. 1514-1530, 2016.

[5] M. Lydon, S. E. Taylor, D. Robinson, A. Mufti, and E. J. O. Brien, "Recent developments in bridge weigh in motion (B-WIM)," Journal of Civil Structural Health Monitoring, vol. 6, no. 1, pp. 69-81, 2016.

[6] F. Moses, "Weigh-in-motion system using instrumented bridges," Journal of Transportation Engineering, vol. 105, no. 3, 1979.

[7] J. Zhang, Y. Lu, Z. Lu, C. Liu, G. Sun, and Z. Li, "A new smart traffic monitoring method using embedded cementbased piezoelectric sensors," Smart Materials and Structures, vol. 24, no. 2, Article ID 025023, 2015.

[8] H. Zhao, N. Uddin, X. Shao, P. Zhu, and C. Tan, "Fieldcalibrated influence lines for improved axle weight identification with a bridge weigh-in-motion system," Structure and Infrastructure Engineering, vol. 11, no. 6, pp. 721-743, 2015.

[9] C. D. Pan, L. Yu, and H. L. Liu, "Identification of moving vehicle forces on bridge structures via moving average Tikhonov regularization," Smart Materials and Structures, vol. 26, no. 8, Article ID 085041, 2017.

[10] Z. Chen, T. H. T. Chan, and A. Nguyen, "Moving force identification based on modified preconditioned conjugate gradient method," Journal of Sound and Vibration, vol. 423, pp. 100-117, 2018.

[11] C.-D. Pan, L. Yu, H.-L. Liu, Z.-P. Chen, and W.-F. Luo, "Moving force identification based on redundant concatenated dictionary and weighted l1-norm regularization," Mechanical Systems and Signal Processing, vol. 98, pp. 32-49, 2018.

[12] H. Sekiya, K. Kubota, and C. Miki, "Simplified portable bridge weigh-in-motion system using accelerometers," Journal of Bridge Engineering, vol. 23, no. 1, Article ID 04017124, 2017.

[13] X. Q. Zhu and S. S. Law, "Recent developments in inverse problems of vehiclebridge interaction dynamics," Journal of Civil Structural Health Monitoring, vol. 6, no. 1, pp. 107-128, 2016.

[14] F. Schmidt, B. Jacob, C. Servant, and Y. Marchadour, "Experimentation of a bridge WIM system in France and applications for bridge monitoring and overload detection," in Proceedings of the International Conference on Weigh-In-Motion ICWIM6, p. 8p, 2012.

[15] R. E. Snyder and F. Moses, "Application of in-motion weighing using instrumented bridges," Transportation Research Record, vol. 1048, pp. 83-88, 1985.

[16] Z.-G. Xiao, K. Yamada, J. Inoue, and K. Yamaguchi, "Measurement of truck axle weights by instrumenting longitudinal ribs of orthotropic bridge," Journal of Bridge Engineering, vol. 11, no. 5, pp. 526-532, 2006.

[17] E. Yamaguchi, S.-I. Kawamura, K. Matuso, Y. Matsuki, and Y. Naito, "Bridge-weigh-in-motion by two-span continuous bridge with skew and heavy-truck flow in Fukuoka area, Japan," Advances in Structural Engineering, vol. 12, no. 1, pp. 115-125, 2009.

[18] Y. Yu, C. S. Cai, and L. Deng, "Nothing-on-road bridge weighin-motion considering the transverse position of the vehicle," Structure and Infrastructure Engineering, vol. 14, no. 8, pp. 11081122, 2018.

[19] Z. Chen, H. Li, Y. Bao, N. Li, and Y. Jin, "Identification of spatio-temporal distribution of vehicle loads on long-span bridges using computer vision technology," Structural Control and Health Monitoring, vol. 23, no. 3, pp. 517-534, 2016.

[20] J. F. Frenze, A Video-Based Method for the Detection of Truck Axles, National Institute for Advanced Transportation Technology, University of Idaho, 2002. 
[21] Y. Lecun, Y. Bengio, and G. Hinton, “Deep learning," Nature, vol. 521, no. 7553, pp. 436-444, 2015.

[22] W. S. Cleveland and S. J. Devlin, "Locally weighted regression: an approach to regression analysis by local fitting," Journal of the American Statistical Association, vol. 83, no. 403, pp. 596610, 1988.

[23] D. A. Freedman, Statistical Models: Theory and Practice, Cambridge University Press, 2009.

[24] W. S. Cleveland, "Robust locally weighted regression and smoothing scatterplots," Journal of the American Statistical Association, vol. 74, no. 368, pp. 829-836, 1979.

[25] A. Znidaric and W. Baumgartner, "Bridge weigh-in-motion systems-an overview," in Proceedings of the Second European Conference on Weigh-In-Motion of Road Vehicles, Lisbon, Portugal, 1998.

[26] P. McNulty and E. J. O'Brien, "Testing of bridge weigh-inmotion system in a sub-arctic climate," Journal of Testing and Evaluation, vol. 31, no. 6, pp. 497-506, 2003.

[27] E. J. OBrien, M. J. Quilligan, and R. Karoumi, "Calculating an influence line from direct measurements," Proceedings of the Institution of Civil Engineers: Bridge Engineering, vol. 159, no. BE1, pp. 31-34, 2006.

[28] S. P. Timoshenko and D. H. Young, Theory of Structures, McGraw-Hill, 1965.

[29] S. P. Timoshenko and J. M. Gere, Mechanics of Materials, van Nordstrand Reinhold Company, New York, NY, USA.

[30] F. Moses, "Instrumentation for weighing truck-in-motion for highway bridge loads," Final Report FHWA-OH-83-001, 1983.

[31] Y. L. Ding, H. W. Zhao, and A. Q. Li, “Temperature effects on strain influence lines and dynamic load factors in a steeltruss arch railway bridge using adaptive FIR filtering," Journal of Performance of Constructed Facilities, vol. 31, no. 4, Article ID 04017024, 2017.

[32] M. Quilligan, Bridge weigh-in motion: development of a 2-D multi-vehicle algorithm [Doctoral thesis], Byggvetenskap, 2003.

[33] J. Schmidhuber, "Deep learning in neural networks: an overview," Neural Networks, vol. 61, pp. 85-117, 2015.

[34] J. Redmon and A. Farhadi, "Yolov3: an incremental improvement," 2018, https://arxiv.org/abs/1804.02767.

[35] G. Xu and Z. Zhang, Epipolar Geometry in Stereo, Motion and Object Recognition: A Unified Approach, Springer, 1996.

[36] H. Kalhori, M. M. Alamdari, X. Zhu, B. Samali, and S. Mustapha, "Non-intrusive schemes for speed and axle identification in bridge-weigh-in-motion systems," Measurement Science and Technology, vol. 28, no. 2, Article ID 025102, 2017.

[37] H. Kalhori, M. M. Alamdari, X. Zhu, and B. Samali, "Nothingon-road axle detection strategies in bridge-weigh-in-motion for a cable-stayed bridge: case study," Journal of Bridge Engineering, vol. 23, no. 8, Article ID 05018006, 2018. 


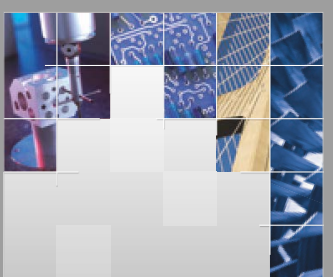

\section{Enfincering}
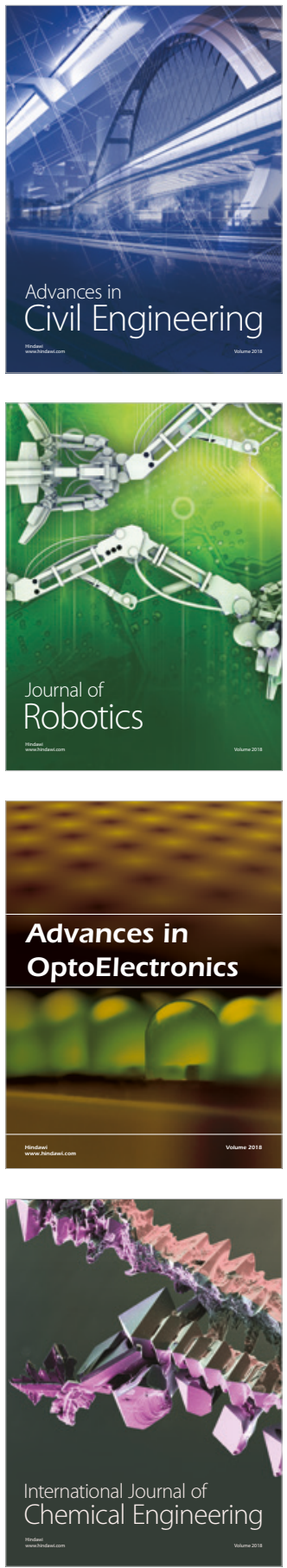

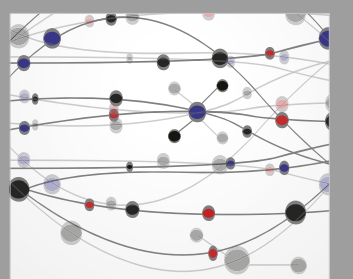

\section{Rotating \\ Machinery}

The Scientific World Journal

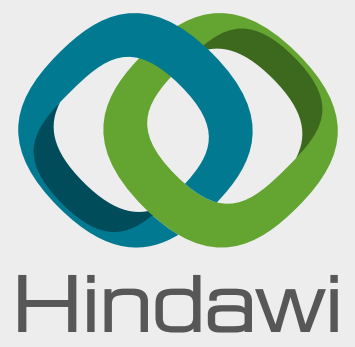

Submit your manuscripts at

www.hindawi.com

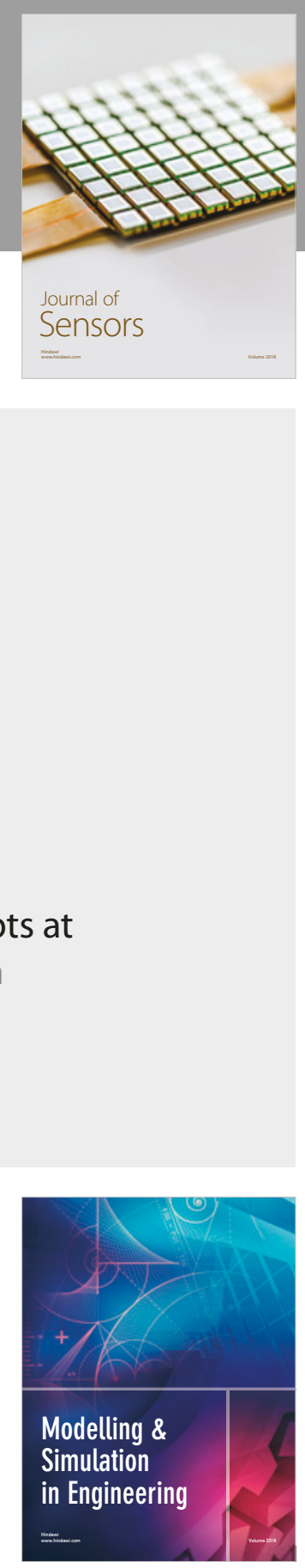

\section{Advances \\ Multimedia}
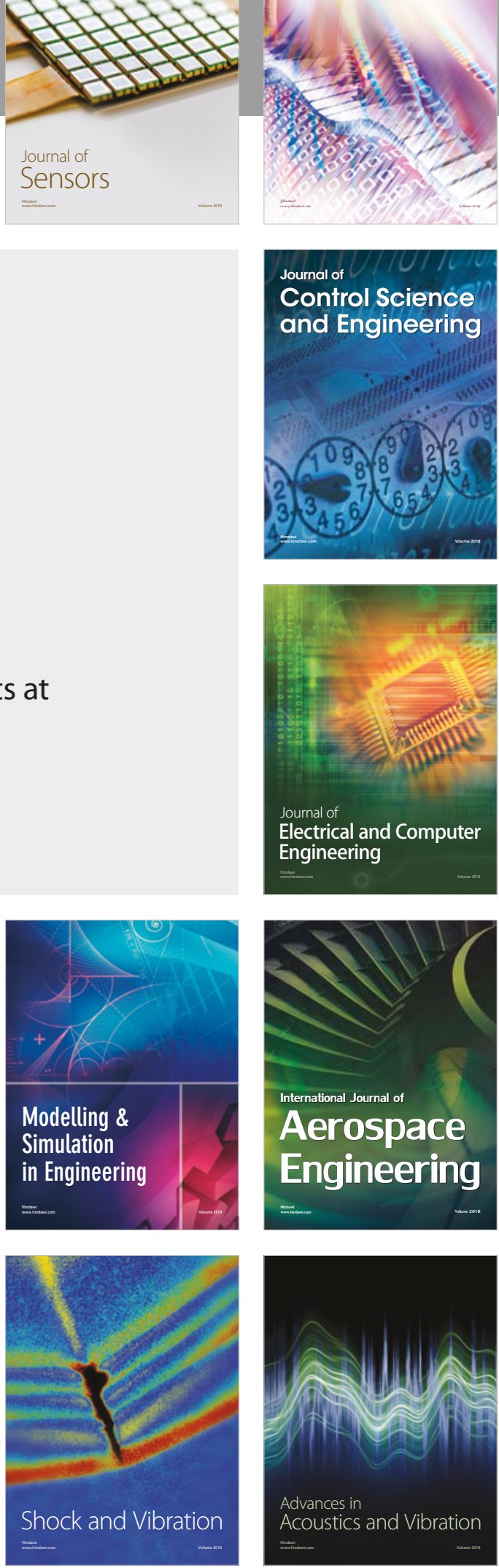